\title{
Simulation research on high-speed motor current harmonic elimination methods
}

\author{
Hong Cui1 ${ }^{1, *}$, and Youqing $\mathrm{Gao}^{2}$ \\ ${ }^{1}$ Liaoning Provincial College of Communications, Shenyang, Liaoning, China \\ ${ }^{2}$ Shenyang Everbright Environment Technology Co., Ltd., Shenyang, Liaoning, China
}

\begin{abstract}
A large number of harmonics will be generated in the process of transforming high frequency and high voltage alternating current (AC) into a constant frequency and constant voltage through a power conversion system when the high-speed motor is used as a generator. The phaseshifting reactor is used to eliminate harmonics for high-speed generator power conversion systems, and LCL filter and adjustable inductance filter are used to eliminate harmonics for high-speed motor power supply system in this paper. The adjustable inductance filter can adjust the inductance value in time according to the change of the output frequency of the inverter. The results verify the effectiveness of the methods used, and provide a theoretical basis for the wide application of high-speed motor by simulation.
\end{abstract}

Keywords: High-speed motor, Phase shift reactor, Filter, Harmonic elimination.

\section{Introduction}

At present, with the invention of new materials, it has become possible to design different types of new motors to accomplish special purposes, and high-speed motors are one of them. It is especially suitable for high-speed machine tool, blower, water pump and other system.

The high-speed generator outputs high-frequency AC, which is transformed into constant-frequency and constant-voltage AC through a power conversion device. In the process of power conversion, the harmonic component is large, which will cause serious interference to the power grid and electrical equipment. The high-frequency inverter is usually used for power supply by high-speed motor. The output current of the inverter contains high-order harmonics, which affects the performance of the high-speed motor ${ }^{[1]}$. Therefore, it is very important to research methods to eliminate the current harmonics of high-speed generator power conversion system and high-speed motor power supply system.

\footnotetext{
*Corresponding author: kate ranran@163.com
} 


\section{Method for eliminating harmonics of high-speed generator power conversion system}

Usually phase-shifting reactor is used as the input transformer in the rectification link of the converter power supply system. The schematic diagram of multi-phase rectification system using phase-shifting reactor is shown in Figure 1.

The impact of these high order harmonics on the power grid can be reduced by using twelve-phase, eighteen-phase or more multi-phase rectifier circuits. The more the number of phases is used in the rectifier circuit, the closer the rectified voltage is to the DC voltage. That is, if a multi-phase rectifier circuit is used for the rectifier device that supplies power to a high-power motor, a DC voltage with less pulsation can be obtained. Reasonable adjustment of the winding turns of the three-phase phase-shifting reactor and phase-tophase switching can obtain the required phase shift angle, which can simultaneously eliminate different orders of harmonics. The simulation study of a twelve-phase rectifier system using a phase-shifting reactor is carried out in this paper. The power supply of the system is a power frequency AC with a phase voltage of $220 \mathrm{~V}$, a load resistance of $200 \Omega$, an inductance of $0.05 \mathrm{H}$, and the best selected phase shift angles of $11^{\circ}$ and $7^{\circ}$.

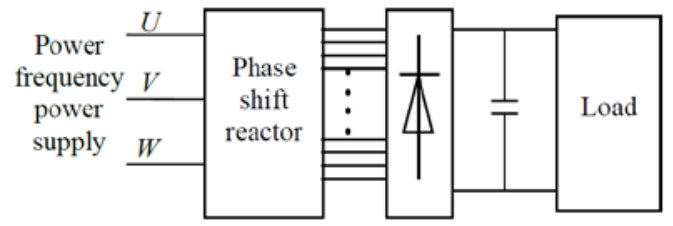

Fig. 1. Schematic diagram of multi-phase rectification system using phase-shifting reactor.

The simulation waveforms of the input current of the three-phase rectification system without the phase-shifting reactor and the input current of the twelve-phase rectification system with the phase-shifting reactor are shown in Figure 2 and Figure 3 respectively.

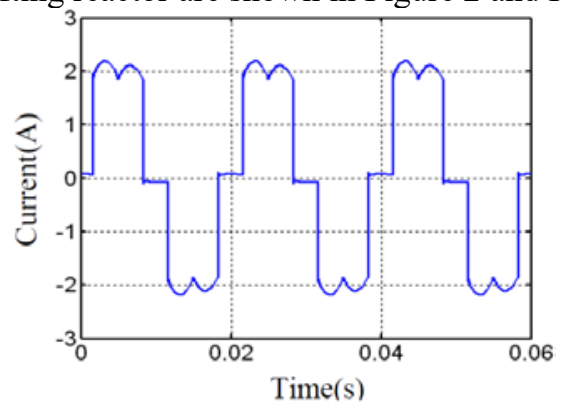

Fig. 2. Input current waveform of three-phase rectifier system without phase-shifting reactor.

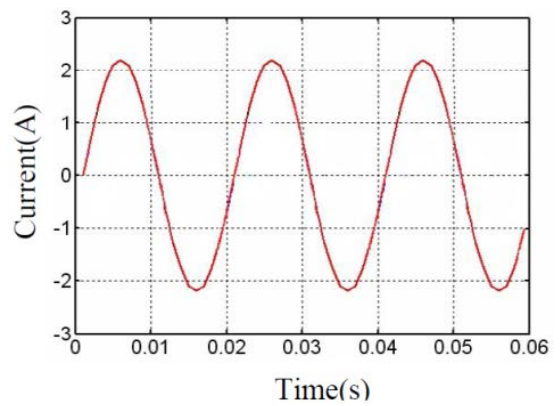

Fig. 3. Input current waveform of twelve-phase rectifier system using phase-shifting reactor. 
It can be seen from Figure 2 and Figure 3 that the multi-phase rectification system using phase-shifting reactors for the high-speed generator power conversion system has a better harmonic elimination effect. The input current is close to a sine wave.

\section{Method for eliminating current harmonics of high-speed motor power supply system}

\subsection{Using PWM frequency converter, the method of eliminating current harmonics of the power supply system when the high-speed motor is at a constant speed}

Due to the use of general high-frequency PWM frequency converters, the harmonic current and voltage of the high-speed motor power supply system are relatively large when the high-speed motor operates as a motor. It will bring adverse effects and affect the stable operation of the high-speed motor ${ }^{[2]}$.

For ordinary motor, LC filters can be used to eliminate the output current harmonics of general PWM frequency converter. However, if the motor speed is extremely high such as tens of thousands of revolutions per minute, the harmonic frequency generated by the PWM inverter is close to the fundamental frequency. So it is difficult to eliminate the harmonics with ordinary LC filter ${ }^{[3]}$.

The LCL filter shown in Figure 4 is used to eliminate the current harmonics of the highspeed motor power supply system in this paper.

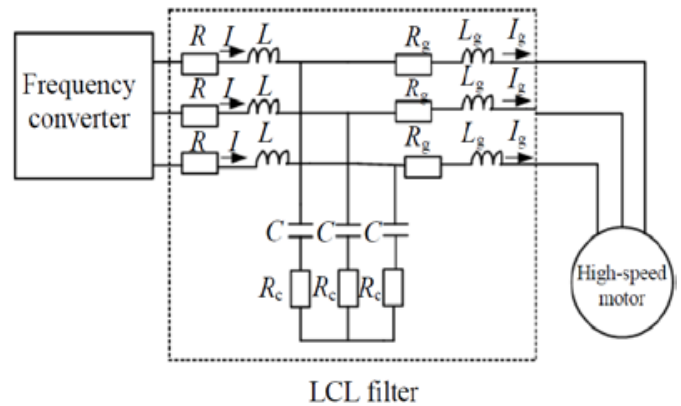

Fig. 4. Schematic diagram of high-speed motor power supply system using LCL filter.

The LCL filter plays the role of injecting compensation current into the high-speed motor power supply system. Compared with the harmonic current, the compensation current is equal in magnitude and opposite in direction, so harmonics can be eliminated.

The controlled object is a high-speed motor with $P_{\mathrm{N}}=10 \mathrm{~kW}, U_{\mathrm{N}}=220 \mathrm{~V}, n_{\mathrm{N}}=12000 \mathrm{r} / \mathrm{min}$, $f_{\mathrm{N}}=200 \mathrm{~Hz}$ in this paper. The parameters of the LCL filter are: $L_{\mathrm{g}}=0.23 \mathrm{mH}, R_{\mathrm{g}}=0.01 \Omega$, $L=0.37 \mathrm{mH}, R=0.03 \Omega, R_{\mathrm{c}}=0.4 \Omega, C=35 \mu \mathrm{F}$, the simulation study of the high-speed motor frequency conversion system is carried out. The simulation waveforms of the current of the high-speed motor power supply system when the filter is not used and the LCL filter is used are shown in Figure 5 and Figure 6 respectively. 


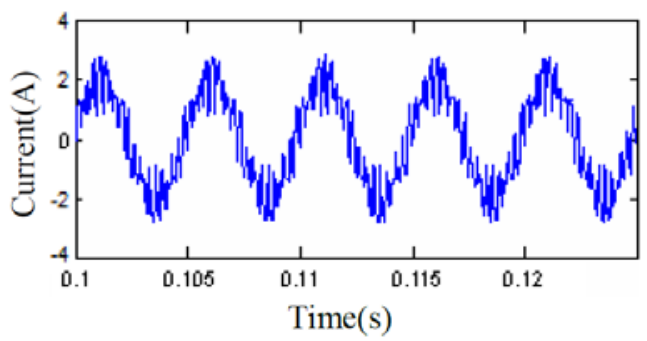

Fig. 5. Current waveform of the high-speed motor power supply system without a filter.

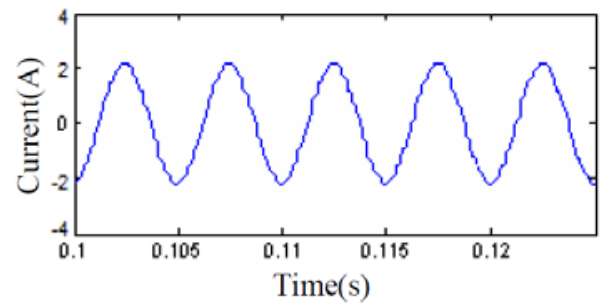

Fig. 6. Current waveform of the high-speed motor power supply system when the LCL filter is used.

It can be seen from Figure 5 and Figure 6 that the LCL filter has a better elimination effect on the current harmonics of the high-speed motor power supply system. The input current is close to a sine wave.

\subsection{The Square Wave frequency converter with adjustable inductance filter is used to eliminate the current harmonics of the power supply system when the high-speed motor changes speed}

If the speed of the high-speed motor is constant, the low-order harmonic current can be reduced by adding a specially designed LC filter at the output of the inverter as described above. However, the LC filter with fixed parameters cannot meet the requirements of the variable speed system because the fundamental frequency and harmonic frequency are variable.

In order to reduce the switching losses of power devices and the harmonics of the inverter output current for high-speed motor at variable speed, a design method of adjustable inductance filter is proposed. The principle block diagram of high-speed motor frequency conversion system and adjustable inductance filter is shown in Figure 7.

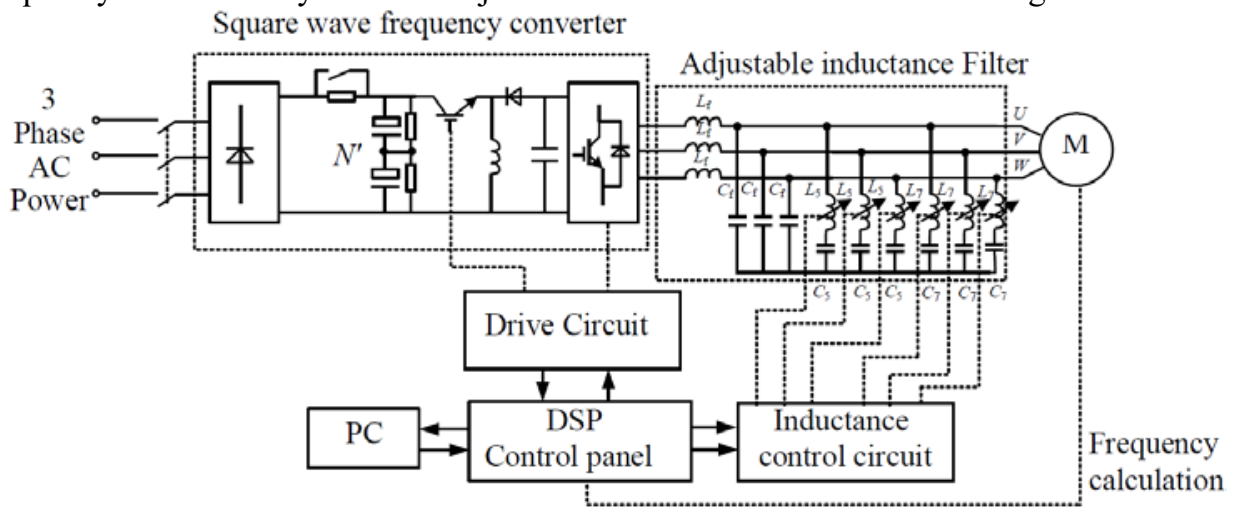

Fig. 7. Principle block diagram of high-speed motor frequency conversion system and adjustable inductance filter. 
The adjustable inductance filter in Figure 7 consists of a low-pass filter and two bandstop filters with adjustable inductance. The low-pass filter is used to reduce high-order harmonics and the band-stop filter is used to eliminate the $5^{\text {th }}$ and $7^{\text {th }}$ harmonics. The bandstop filter can adjust the bandwidth with the change of the frequency of the inverter output current. The parameters of the band-stop filter depend on the frequency of the harmonics to be eliminated and the LC series resonance conditions. The parameters of adjustable inductance can be changed with frequency. The same control unit is used to control the frequency converter and filter.

The schematic diagram of the winding connection of the adjustable inductor is shown in Figure 8. The two AC windings wound on the iron cores on both sides are connected in reverse series, and the DC excitation winding is wound on the middle iron core. The direction of the magnetic flux generated by the two AC winding currents on the middle iron core is always opposite. Since the inductance increments of the two AC windings are equal and opposite, no AC induced voltage is generated in the DC excitation winding ${ }^{[4]}$.

The inductance value can be changed by controlling the DC excitation current, so that the adjustable band-stop filter works at the resonance point. The change range of adjustable inductance $L_{5}$ and $L_{7}$ is determined by the change range of the high-speed motor speed. The inductances $L_{5}$ and $L_{7}$ cannot be too large, otherwise the voltage drop on the inductance will be too large and the power supply voltage of the high-speed motor will be too small, and the motor will not start.

For the system shown in Figure 7, the above-mentioned high-speed motor is used as the controlled object, and the output terminal of the square wave frequency converter is simulated and studied in different cases with no filter added and an adjustable inductance filter added. In these two cases, the simulation results of the current waveforms of the highspeed motor power supply system are shown in Figure 9 and Figure 10 respectively.

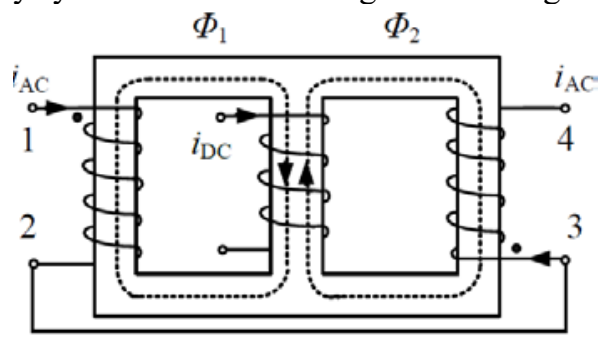

Fig. 8. Schematic diagram of winding connection of adjustable inductor.

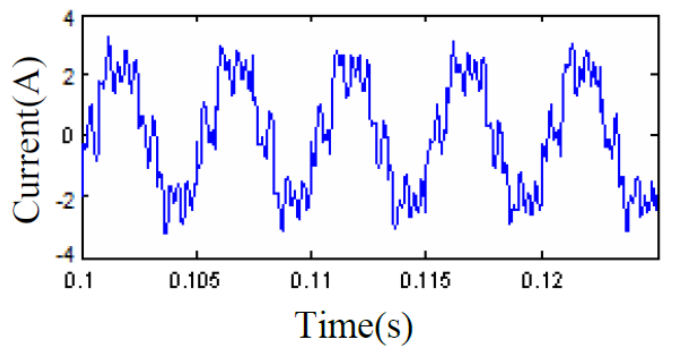

Fig. 9. Current waveform without filter $(f=200 \mathrm{~Hz})$. 


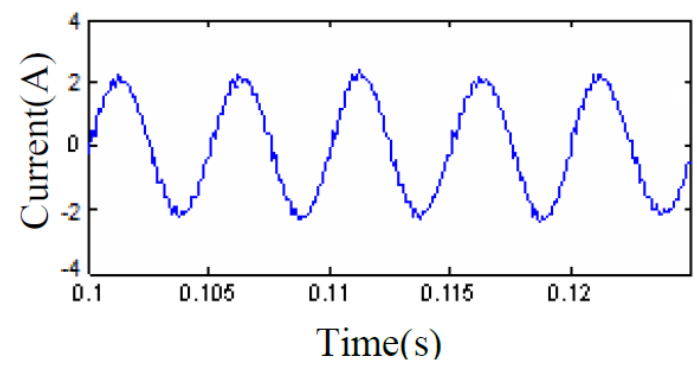

Fig. 10. Current waveform with adjustable inductance filter $(f=200 \mathrm{~Hz})$.

It can be seen from Figure 9 and Figure 10 that the current harmonics of the high-speed motor power supply system are very large when the filter is not added. After adding an adjustable inductance filter at the output of the frequency converter, the current of the highspeed motor power supply system is close to a sine wave. It can be seen that using a square wave frequency converter and adding an adjustable inductance filter at the output of the frequency converter is a better way to reduce current harmonics in the power supply system of a variable speed high-speed motor.

\section{Conclusion}

It can be seen from the simulation for the input current of the high-speed generator power conversion system and the current of the high-speed motor power supply system:

(1) When a high-speed motor is used as a generator, the multi-phase rectification system using phase-shifting reactors for the high-speed generator power conversion system has a good elimination effect on harmonics, and the input current is close to a sine wave.

(2) When a high-speed motor is used as a motor, a PWM frequency converter is used. When the high-speed motor is at a constant speed, the current harmonics of the power supply system can be eliminated by using an LCL filter on the output side of the frequency converter.

(3) The proposed adjustable inductance filter is an effective method to reduce switching losses and current harmonics for variable speed high speed motor power supply systems.

\section{References}

1. Lascu C, Asiminoaei L, Boldea I, Blaabjerg F. Frequency Response Analysis of Current Controllers for Selective Harmonic Compensation in Active Power Filters. IEEE Trans. on Industrial Electronics, 2009, 56(2), p 337-347.

2. O. Aglen, A. Anderson. Thermal Analysis of a High-speed Generator. Proc. of IEEEIAS Annual Meeting, 2003, p 547-554.

3. Li Liyi, Tan Guangjun, Liu Jiaxi, Kou Baoquan. Inductance-capacitance Filter Circuit for Reducing Current Harmonics of High-speed Motor. Micromotors, 2013, 46(7), p $38-44$.

4. Chen Xiyou, Yan Bin, Xu Dianguo, Ma Hongfei. Principle and Practice of Adjustable Inverter Output Filter. Journal of Harbin Institute of Technology, 2003, 35(5), p 549552. 